\title{
Thickness-Dependent Resonant Raman and E' Photoluminescence Spectra of Indium Selenide and Indium Selenide/Graphene Heterostructures
}

Srinivasa Reddy Tamalampudi, $\stackrel{\dagger}{\dagger}$ Raman Sankar, $"$ Harry Apostoleris, ${ }^{\dagger}+$ Mariam Ali Almahri,

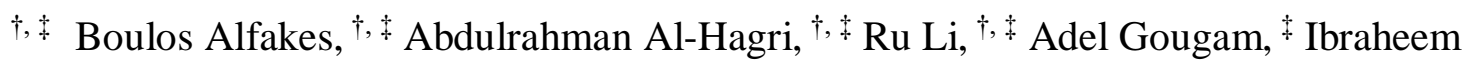
Almansouri, ${ }^{\dagger}$ Matteo Chiesa, ${ }^{*},+,+$, Jin-You Lu ${ }^{*}, \dagger, *$

$\dagger$ Laboratory for Energy and NanoScience (LENS), Khalifa University of Science and Technology, Masdar Campus, PO Box 54224, Abu Dhabi, UAE

\$Depatment of Mechanical and Materials Engineering, Khalifa University of Science and Technology, Masdar Campus, PO Box 54224, Abu Dhabi, UAE

" Institute of Physics, Academia Sinica, Taipei R.O.C. 11529, Taiwan

+ Department of Physics and Technology, UiT The Arctic University of Norway, 9010 Troms $\varnothing$, Norway

*matteo.chiesa@ku.ac.ae, *chun.lu@ku.ac.ae 


\begin{abstract}
Atomically thin, two-dimensional (2D) indium selenide (InSe) has attracted considerable attention owing to the dependence of its bandgap on sample thickness, making it suitable for small-scale optoelectronic device applications. In this work, by the use of Raman spectroscopy with three different laser wavelengths, including $488 \mathrm{~nm}, 532 \mathrm{~nm}$, and $633 \mathrm{~nm}$, representing resonant, near-resonant and conventional non-resonant conditions, a conclusive understanding of the thickness dependence of lattice vibrations and electronic band structure of InSe and InSe/graphene heterostructures are presented. Combining our experimental measurements with first-principles quantum mechanical modeling of the InSe systems, we identified the crystal structure as $\varepsilon$-phase InSe and demonstrated that its measured intensity ratio of Raman peaks in the resonant Raman spectrum evolves with the number of layers. Moreover, graphene coating enhances Raman scattering of few-layered InSe and also makes its photoluminescence stable under higher-intensity laser illumination. The optically induced charge transfer between van der Waals graphene/InSe heterostructures is observed under excitation of the E' transition in InSe, where the observed mechanism may potentially be a route for future integrated electronic and optoelectronic devices.
\end{abstract}




\section{Introduction}

Recently, several III-VI group van der Waals layered structures, such as $\mathrm{GaS},{ }^{1} \mathrm{GaSe},{ }^{2}$ $\mathrm{GaS}^{3}{ }^{\text {and }} \mathrm{InSe}{ }^{4-6}$ and their heterostructures have been considered as potential materials for next-generation optoelectronic devices owing to their superior electrical and optical properties. Among these layered structures, multilayer InSe field effect transistors show greater potential since they can absorb the light through the visible spectrum and yield very high electron mobility of $0.1 \sim 2.0 \times 10^{3} \mathrm{~cm}^{2} / \mathrm{VS}$ at ambient conditions. ${ }^{7,8}$ Several studies on $2 \mathrm{D}$ semiconductor layered structures have shown that their electronic and optical properties depend on the thickness and layer orientation or phase of the materials. ${ }^{9,10}$ Layered InSe exists in at least three different crystallographic structures based on the stacking order variation among the individual InSe layers, ${ }^{11}$ which exhibit different electronic band structures and corresponding optoelectrical response. $^{12}$ For instance, the $\gamma$ phase (space group $C_{3 V}^{5}$ ) InSe corresponds to a rhombohedral structure, and both of $\beta$ phase ( $\operatorname{space}$ group $D_{6 h}^{4}$ ) and $\varepsilon$ (space group $D_{3 h}^{1}$ ) corresponds to hexagonal structures, with a slightly different stacking sequence. $\beta$-InSe possesses an inversion symmetric center, ${ }^{13}$ while $\varepsilon$-InSe is non-centrosymmetric. ${ }^{14,15}$

In this work, we perform X-ray diffraction (XRD) and transmission electron microscopy (TEM) measurements to explore the crystallographic structure of InSe bulk crystals grown using the Bridgman method. ${ }^{16}$ The InSe samples, ranging in thickness from bulk to a few layers were further characterized using non-resonant and resonant Raman spectroscopy. ${ }^{17}$ As compared to non-resonant (common) Raman spectroscopy, the resonant Raman spectroscopy is carried out using a laser with energy near the electronic transition of materials, which is more sensitive to 
the atomic ordering of the crystal structure. The resonant Raman spectra exhibit additional Raman modes that are normally forbidden in the standard (non-resonant) Raman spectra, which provides additional information on crystal structure, ${ }^{18}$ including crystal phase, defects, dopants, and strains. In parallel, first-principles density functional theory (DFT) simulation was carried out to explore phonon modes of InSe from bulk to few layers, including infrared and Raman active modes under non-resonant conditions. A combination of resonant Raman spectroscopy and DFT predicted lattice dynamics of InSe from bulk to few layers helps identifying the crystal phase and thickness of InSe.

The absorption spectrum of InSe is characterized by three peaks in the wavelength range between $400 \mathrm{~nm}$ and $1000 \mathrm{~nm},{ }^{19}$ which correspond to three photoexcited electron-hole pair generations, including band gap transition $(\sim 1.2 \mathrm{eV}), \mathrm{E}^{\prime}(\sim 2.4 \mathrm{eV})$, and $\mathrm{E}^{\prime \prime}(\sim 2.9 \mathrm{eV})$ transitions, ${ }^{19}$ respectively. The $\mathrm{E}^{\prime}$ transition is the energy difference between the lowest conduction band and second highest valence band. In contrast to $\mathrm{MoS}_{2}$ and $\mathrm{WS}_{2},{ }^{20}$ the photoluminescence (PL) of the $\mathrm{E}^{\prime}$ transition of InSe is stronger than that of its band gap emission owing to the larger absorption coefficient of the $E^{\prime}$ transition. ${ }^{21}$ In this work, the thickness dependence of InSe on its $\mathrm{E}^{\prime}$ transition induced PL spectrum is investigated and the characterization results are compared with DFT predicted electronic band structures.

Previous study has indicated that InSe rapidly degrades at ambient conditions, ${ }^{22,23}$ and during the experiment, we observe similar phenomenon, particularly for thinner InSe flakes, which is possibly due to the increased Se vacancies causing quicker absorption of oxygen and moisture. ${ }^{22}$ The degradation is one of the major issues among $2 \mathrm{D}$ material based devices. ${ }^{24}$ To keep atomically thin InSe stable for long-term and practical applications, we transfer CVDgrown graphene onto InSe flakes as a protective capping layer using a wet-transfer process. Our 
characterization results show that graphene coating enhances the Raman scattering of InSe and makes the PL signal of InSe more stable. The dependence of graphene layer thickness (1L, 2L and ML) on the InSe/graphene heterostructure is also investigated using resonant Raman and PL spectroscopy measurements. Moreover, graphene coating not only works as a protection layer, but also acts as a heterostructure with InSe. Our experimental and theoretical studies indicate that under laser illumination, the E' transition-induced electrons are injected into the graphene from the InSe. Therefore, in combining recent studies on InSe/graphene heterojunction structures such as high broad band photo detector ${ }^{25}$ and gate tunable transistors, ${ }^{26}$ the InSe/graphene heterostructure shows great application potential in downscaling optoelectronic devices.

\section{Experimental Section:}

Crystal growth

The growth method and parameters of InSe crystals were described in previously reported studies ${ }^{4}$. Briefly, the molar mixture of In $(52.4 \%)$ and Se $(47.6 \%)$ compounds is placed in a conical quartz ampoule and then evacuated to $10^{-4} \mathrm{~Pa}$. The horizontal furnace temperature is held at $550^{\circ} \mathrm{C}$ for $48 \mathrm{~h}$ for homogenization of the batches and synthesis of the InSe flakes and vertical Bridgman method used to grow the mixed crystals. The melt was heat-treated at $850^{\circ} \mathrm{C}$ for $24 \mathrm{~h}$ and then quartz ampoule was cooled down through a temperature gradient of $1^{\circ} \mathrm{C}$ at a rate of 0.1 $\mathrm{mm} / \mathrm{h}$.

\section{Material characterization}

The lattice parameters and crystal structure of as-grown InSe crystals are probed by X-ray diffraction (XRD) using a X'Pert PRO-PANalytical, CuKa radiation. The mechanical exfoliation of the bulk InSe crystal yields various thicknesses of InSe flakes ranging from many-layer to 
single-layer and transfer to the cleaned $\mathrm{Si} / \mathrm{SiO}_{2}$ substrate for further Raman characterizations. The non-resonant, near-resonant and resonant Raman spectrum characterizations were carried out with WITEC 300s with 633, 532 and $488 \mathrm{~nm}$ laser excitations respectively and a spot size of $\sim 10 \mu \mathrm{m}$. The liquid exfoliation of bulk InSe in isopropyl alcohol (IPA) yields few layer thin InSe flakes, which were transferred onto the TEM grid (holey carbon on copper grid with 300 mesh from Electron Microscopy Sciences) for further analysis.

\section{Graphene growth and transfer}

The chemical vapor deposition of graphene has been described in our previous work. ${ }^{27}$ The $\mathrm{Cu} / \mathrm{graphene}$ stack was covered with PMMA and the $\mathrm{Cu}$ foil was subsequently etched away by iron chloride $\left(\mathrm{FeCl}_{3}\right)$ about 60 mins. Thereafter, the PMMA/graphene stack was cleaned with deionized water 3-5 times, then transferred onto the exfoliated InSe flakes on $\mathrm{SiO}_{2} / \mathrm{Si}$ substrate and dried at ambient conditions about 24 hours. Then, the PMMA was dissolved in acetone, and the sample was cleaned with IPA and dried with nitrogen gas.

\section{Computational Section:}

\section{Phonon frequency and intensity of Density functional theory simulation}

All DFT phonon frequency and intensity studies were performed using the Quantum Espresso package. ${ }^{28}$ We adopted norm-conserving pseudopotentials, and the exchange-correlation functional is in the form of local-density approximation (LDA) with parametrization of PerdewZunger. ${ }^{29}$ The kinetic energy cutoffs and density cutoffs are set as $80 / 320$ Ry with $24 \times 24 \times 8$ Monkhorst-Pack k-point mesh for both $\varepsilon$-InSe and $\beta$-InSe. The phonon frequencies are calculated at the gamma point using density-functional perturbation theory, and the IR and Raman cross sections are calculated based on ref. ${ }^{30}$ 


\section{Electronic band structure of Density Functional Theory Simulations}

The DFT calculations were carried out using the VNL-ATK ${ }^{31}$ with the GGA-DFT+1/2 $2^{32}$ exchange functional. The density mesh cutoff is $400 \mathrm{Ry}$, and the Brillouin zone is sampled with a grid of $5 \times 5 \times 1 \mathrm{k}$-points within the Monkhorst-Pack scheme. Before calculating the band structure, all atomic positions of InSe were fully relaxed until the Hellmann-Feynman force on each ion and total energy change are $<0.05 \mathrm{eV} \AA^{-1}$ and $1 \times 10-4 \mathrm{eV}$, respectively. The spin-orbit coupling is included in our electronic band structure calculations.

\section{Results \& Discussions}

\section{Part 1: Thickness dependent lattice dynamics from bulk to few layers of InSe}

The schematic depiction of InSe crystal structure is shown in Fig. 1(a). Every single layer of InSe contains two planes of In metal atoms sandwiched between two planes of Se atoms with the arrangement Se-In-In-Se. These planes are bound together by covalent bonds, and interactions among the InSe layers are weak van der Waals forces, which allow InSe flakes to easily be exfoliated using the scotch tape method. Previous literature ${ }^{33}$ showed that InSe exists in three phases: hexagonal $\beta$ phase, rhombohedral $\gamma$ phase and hexagonal $\varepsilon$ phase. The crystal structure of our bulk sample is characterized using TEM, as shown in figure 1(b). From the TEM image of InSe and its selected area electron diffraction pattern analysis (Figure 1b inset), it is clear that the sample has a hexagonal structure, and thus it belongs to either the $\beta$ or $\varepsilon$ phase. Additional crystallographic measurement has been done by XRD, and the retrieved lattice

constants are $\mathrm{a}=\mathrm{b}=4.005 \AA$ and $\mathrm{c}=16.64 \AA$, which is included in Fig. S1 of Supplementary Material. However, as the $\beta$ and $\varepsilon$ phases have both the same crystallographic structure and 
similar lattice constant,${ }^{34}$ crystallographic measurements are of limited use in distinguishing these two phases.

(a)

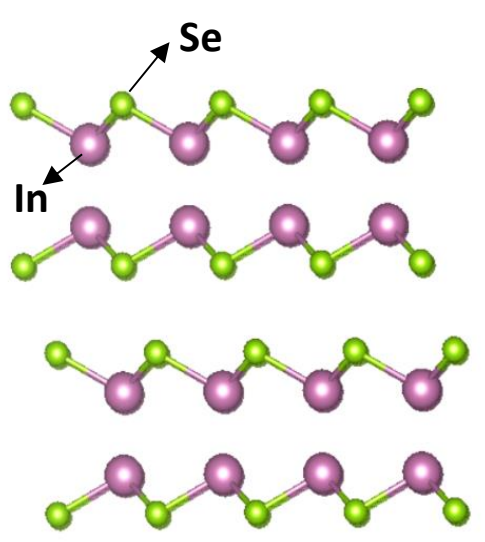

(c)

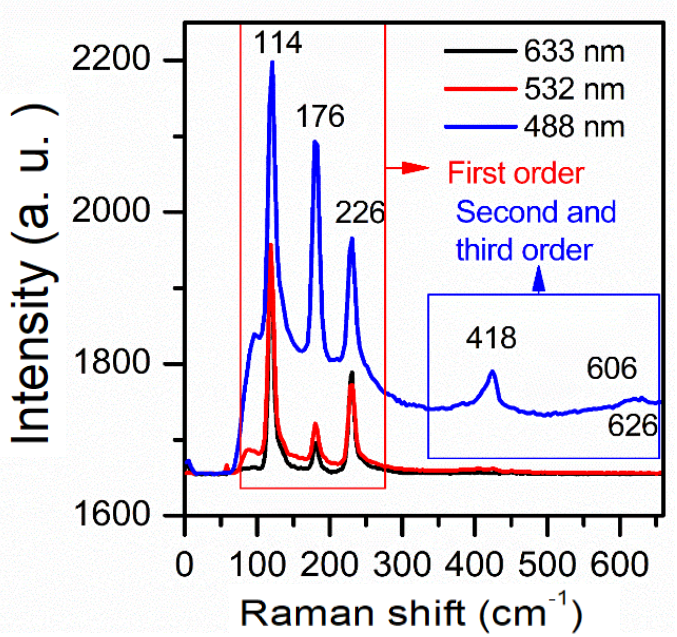

(b)

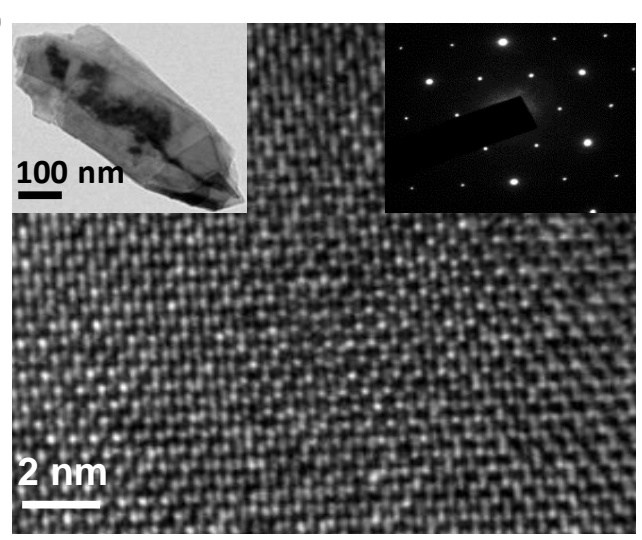

(d)

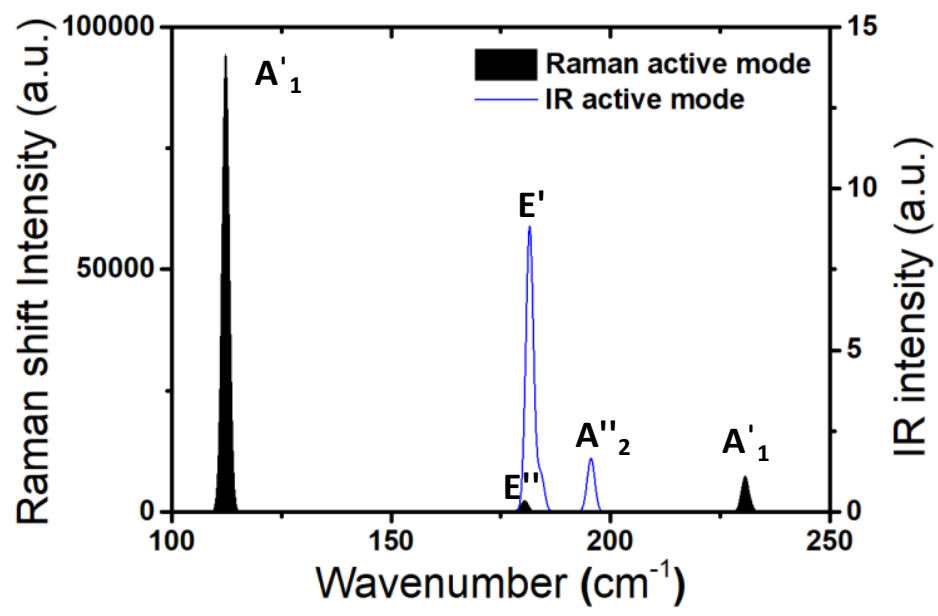

Figure 1. (a) Structure of $\varepsilon$-InSe. (b) High-resolution TEM image of InSe sample and its hexagonal electron diffraction pattern. (c) Raman spectra of bulk InSe under non-resonant, nearresonant, and resonant conditions. (d) DFT predicted Raman and IR spectra of bulk $\varepsilon$-InSe.

We, therefore, employ the standard technique of Raman spectroscopy to explore the phase of bulk InSe, which has a few hundreds of micrometer thickness. In Figure 1(c), we display the Raman spectra of the sample for three excitation wavelengths, $633 \mathrm{~nm}, 532 \mathrm{~nm}$, and $488 \mathrm{~nm}$, respectively. Among three laser wavelengths, the excitation wavelength of $488 \mathrm{~nm}$ (2.54 
eV) has higher energy to effectively excite PL emission, which is not induced by the lasers of $532 \mathrm{~nm}$ and $633 \mathrm{~nm}$. According to the applied laser energies, we consider the three excitation wavelengths as three conditions of non-resonant $(633 \mathrm{~nm})$, near-resonant $(532 \mathrm{~nm})$ and resonant (488 nm), respectively. All Raman spectra show three first-order Raman-active peaks at $114 \mathrm{~cm}^{-1}$, $176 \mathrm{~cm}^{-1}$, and $226 \mathrm{~cm}^{-1}$. In addition to the excitation of first-order lattice phonons, the $488 \mathrm{~nm}$ laser also induces additional second and third order lattice vibrational modes. This is because the laser energy of $488 \mathrm{~nm}$ is close in energy to the E' electronic transition of InSe, where the energy transition is the energy difference between the second highest valence band to lowest conduction band. This frequency coincidence causes the coupling between E' exciton and lattice phonons, which further enhances the intensity of Raman scattering and leads to the appearance of additional peaks in the Raman spectrum as seen on the right-hand side of Figure 1(c). Details of electronic properties will be discussed in the next section.

In order to give more insight into the Raman characterization results, we perform firstprinciples density functional theory simulation using the Quantum-ESPRESSO package ${ }^{28}$ to investigate the lattice dynamics of bulk InSe. It should be noted that the resonant condition of lattice dynamics is not included in the DFT simulation due to the limitations of current implementations of Quantum-ESPRESSO package for excited states. Detailed parameters of input files for phonon calculations are included in the Supplementary Material. The frequencies and intensities of phonon modes of bulk $\varepsilon$-InSe and $\beta$-InSe, including infrared (IR) and Raman active modes, are calculated and plotted in the spectra, as shown in Fig. 1(d) and Fig. S2(a). The DFT-predicted Raman shift spectra of the $\varepsilon$-InSe show three Raman active modes, including $\mathrm{A}_{1}$ $\left(112 \mathrm{~cm}^{-1}\right), E^{\prime \prime}\left(178.5 \mathrm{~cm}^{-1}\right)$, and $\mathrm{A}_{1}^{\prime}\left(231.5 \mathrm{~cm}^{-1}\right)$, which agree with the experimentally measured Raman modes. These peaks are vibration modes with the symmetry of $\mathrm{A}_{1}^{\prime}, \mathrm{E}^{\prime \prime}$, and $\mathrm{A}^{\prime}{ }_{1}$, 
respectively, based on group-theoretical consideration on the space group $\mathrm{D}_{3 \mathrm{~h}}$ of $\varepsilon$-InSe. The " $\mathrm{A}$ " symmetry modes correspond to the out-of-plane atomic displacements, and the E symmetry modes are "in-plane" atomic displacements. In addition to three Raman active modes, two polar phonons modes, including $\mathrm{E}^{\prime}\left(182 \mathrm{~cm}^{-1}\right)$ and $\mathrm{A}_{2}^{\prime}\left(196 \mathrm{~cm}^{-1}\right)$ are predicted in the IR absorption spectra. However, there is no significant difference of phonon modes, including frequencies and intensity, in bulk InSe between $\varepsilon$ and $\beta$ phases, as indicated by the DFT simulation. Therefore, we cannot distinguish the crystal phase from characterizing the bulky InSe sample.

We proceed to obtain a few-layer flake using mechanical exfoliation on $\mathrm{SiO}_{2} / \mathrm{Si}$ wafer. The Raman measurements are repeated at all three wavelengths. As shown in Figure 2(a), the resonant Raman spectra of the few-layer flake now exhibit a strong peak at $200 \mathrm{~cm}^{-1}$, which is a marker of the $\varepsilon$ phase, as indicated by earlier works on InSe $\cdot{ }^{11,34}$ In our work, we found that $\mathrm{A}_{2}{ }_{2}$ $\left(200 \mathrm{~cm}^{-1}\right)$ peak is thickness-dependent, and it is not observed in the micrometer-thick InSe. Meanwhile, in comparison of the Raman characterization results of few-layered InSe, we perform DFT simulation to calculate the frequencies and intensities of phonon modes of bilayered $\varepsilon$-InSe and $\beta$-InSe, as shown in Fig. 2(b) and Fig. S2(b), respectively. According to DFT simulation results, the $\mathrm{A}_{2}^{\prime}\left(200 \mathrm{~cm}^{-1}\right)$ peak corresponds to polar IR active mode, which is forbidden in the non-resonant Raman spectra. More importantly, DFT simulation results show that the $\mathrm{A}_{2}^{\prime}\left(200 \mathrm{~cm}^{-1}\right)$ IR-active peak is not existed in the phonon modes of bilayered $\beta$-InSe due to its center symmetry, as shown in Fig. S2(b). Therefore, we confirmed the crystal phase under the study as $\varepsilon$-phase. The lattice vibrations of these observed Raman modes are included in Fig. S3 of Supplementary Materials for reference. 
In Figure 2(c), we investigate the evolution of the resonant Raman spectrum (488 nm laser) with changing InSe thickness from $90 \mathrm{~nm}$ to $8 \mathrm{~nm}$. It is important to note that, the exfoliated few layer thick InSe flakes are not stable at ambient conditions. However, the degradation of the InSe is not as much as rapid than the black phosphorous and $\mathrm{GaSe}{ }^{35,36}$ It has been reported that a 4 layer InSe flake took almost 5 days to degrade completely at ambient conditions. ${ }^{34}$ In the current study, we carried out all the measurements on the same day as soon as the InSe flakes were prepared to avoid the degradation effect on characterization results. The $\mathrm{A}_{2}^{\prime}\left(200 \mathrm{~cm}^{-1}\right)$ peak is small for the thickest $90 \mathrm{~nm}$ flake and becomes more prominent as the thickness is reduced to $8 \mathrm{~nm}$. We furthermore note the small shift in the Raman peaks with decreasing thickness. 


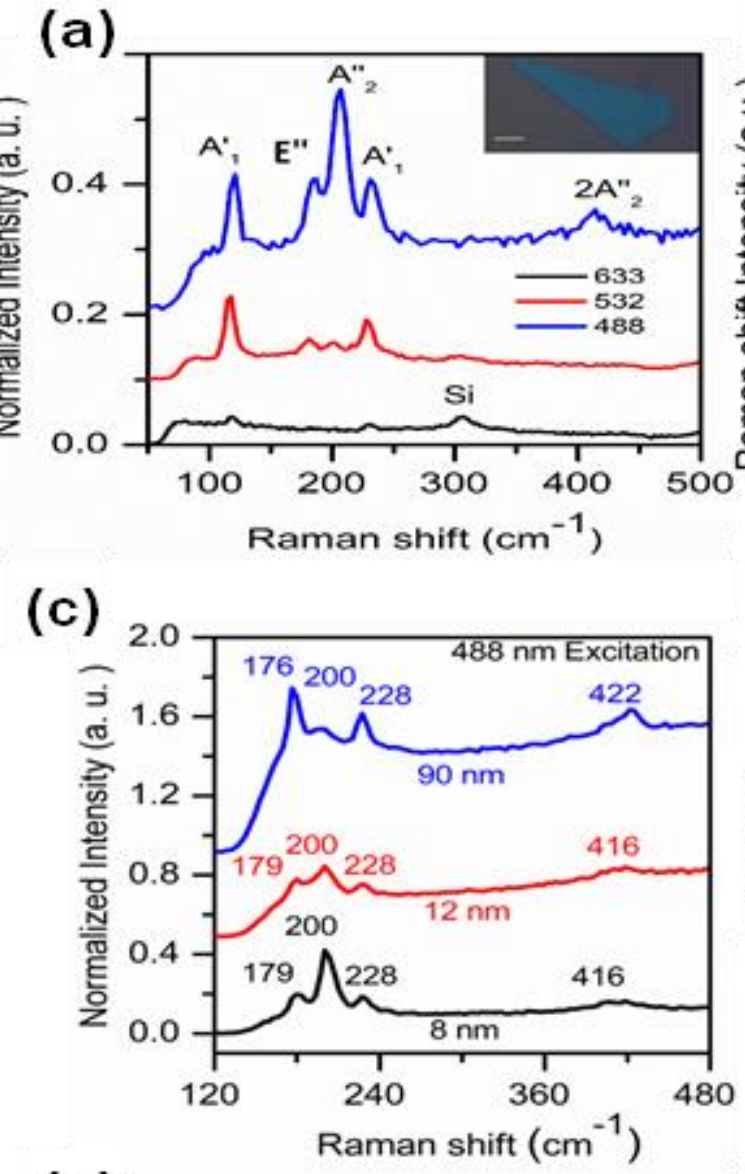

(b)

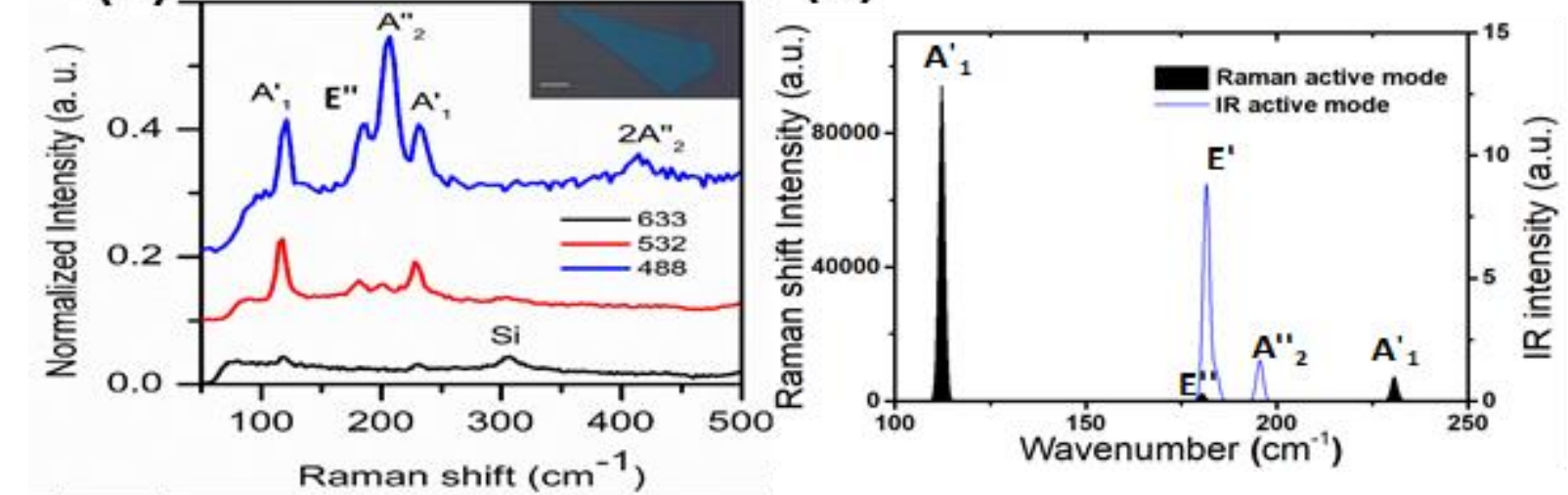

(c)
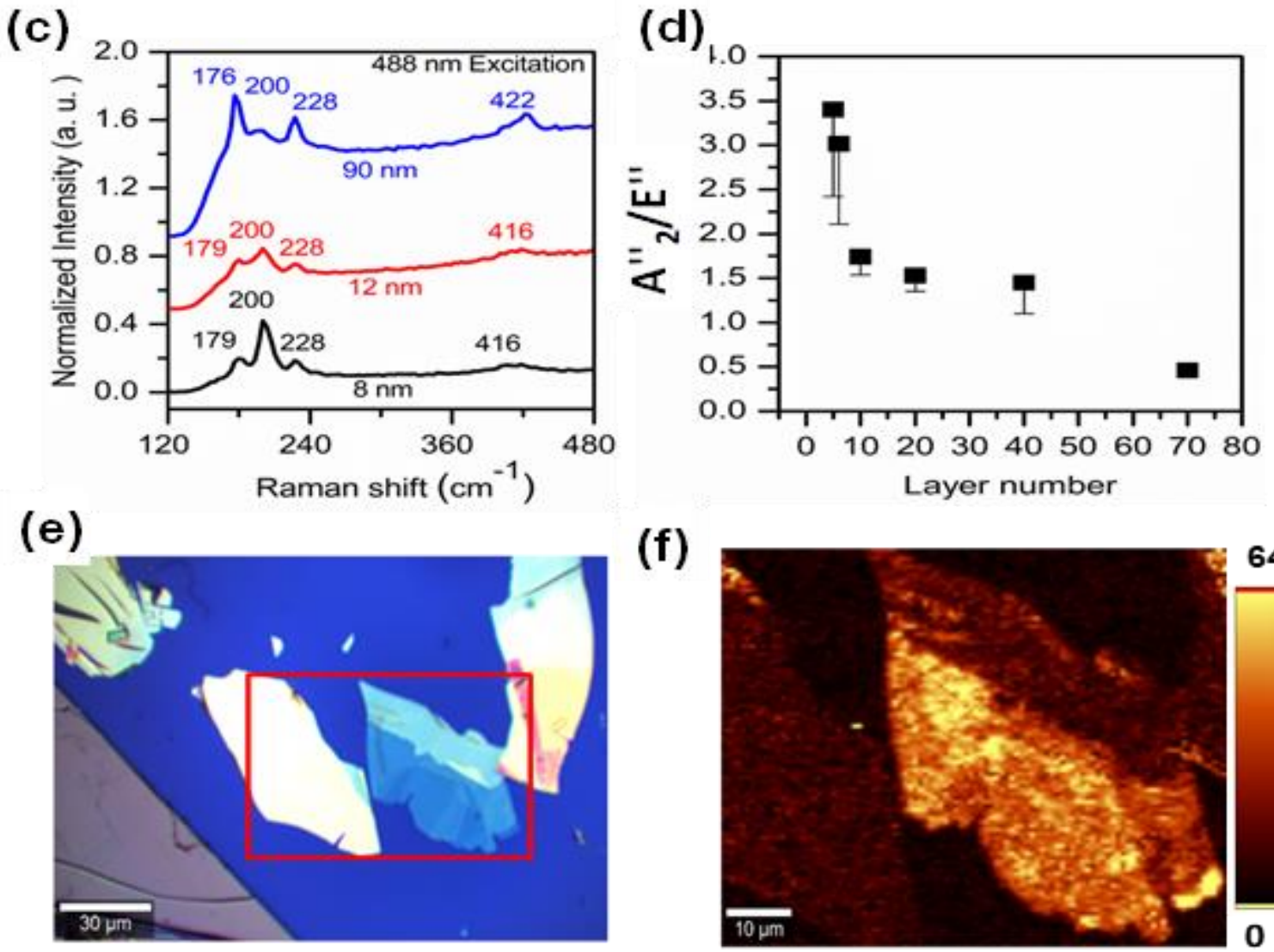

(f)

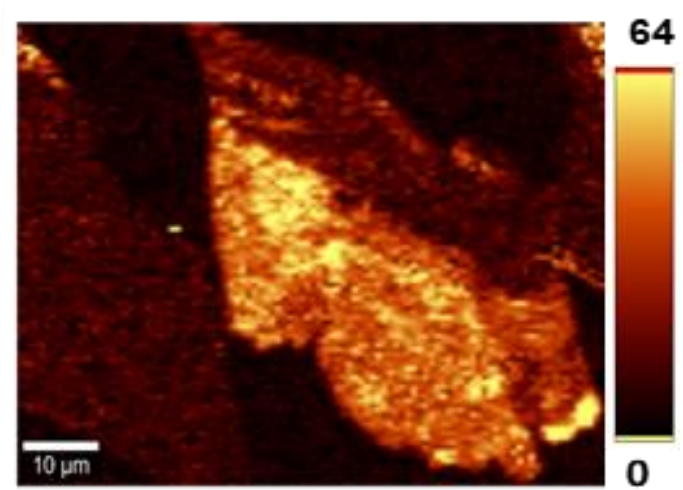

Figure 2. (a) Raman spectra of few-layered InSe under the illumination of three different lasers, including $488 \mathrm{~nm}, 532 \mathrm{~nm}$, and 633nm, respectively. (b) DFT predicted Raman and IR spectra of bilayered $\varepsilon$-InSe. (c) Resonant Raman spectra of InSe with different layers at the excitation of $488 \mathrm{~nm}$ laser. (d) Intensity ratio of $\mathrm{A}_{2}^{\prime} / \mathrm{E}^{\prime \prime}$ peak in the resonant Raman spectra of InSe with 
different layer number. (e) An optical image of InSe with different number of layers. (f) Raman mapping of $\mathrm{A}_{2}\left(200 \mathrm{~cm}^{-1}\right)$ peak intensity on the same region, as highlighted in the optical image.

This shift of the $\mathrm{A}_{2}^{\prime}\left(200 \mathrm{~cm}^{-1}\right)$ peak has previously been used to identify InSe flake thickness ${ }^{12}$ however, the far more pronounced change in the peak intensities that we observe potentially represents a more reliable way to relate the resonant Raman characteristics to the sample thickness. In Fig. 2(d) we plot the measured relationship between sample thickness and the peak ratios of $\mathrm{A}_{2}^{\prime} / \mathrm{E}^{\prime \prime}$ peaks, according to the notation in Fig. 2(a). The plot shows a strong increasing trend of peak ratio as the thickness is decreased down to 5 6 layers, representing the increase of the $\mathrm{A}_{2}^{\prime}\left(200 \mathrm{~cm}^{-1}\right)$ peak with decreasing thickness. An area of InSe flakes with different layer number, as shown in Fig. 2(e), is chosen for performing Raman mapping characterization. In the Raman mapping of Fig.2 (f), the $\mathrm{A}_{2}^{\prime}\left(200 \mathrm{~cm}^{-1}\right)$ peak intensity is increased with decreasing the thickness in comparison with Fig.2 (e). Therefore, our study suggests that the intensity ratio of Raman $\mathrm{A}_{2}^{\prime} / \mathrm{E}^{\prime \prime}$ peaks under the resonant condition can be used to identify the thickness of InSe flakes. It is interesting to look into the Raman spectra of monolayer, or bilayer of InSe; however, we did not observe any signals from them, as shown in Fig. S5(b-d).

The observation of $\mathrm{A}_{2}^{\prime}\left(200 \mathrm{~cm}^{-1}\right)$ peak on InSe in the resonant Raman spectroscopy is possibly owing to two factors: one is the interaction between E' transition electron-hole pair generation and polar phonons. ${ }^{37}$ The other is that the intensity of $\mathrm{A}_{2}^{\prime}$ mode is related to light absorption of InSe, which is stronger in the thinner flakes, as indicated by the color of InSe flakes in the optical image (Fig. 2(e)). These effects cause the out-of-plane IR-active (and Raman forbidden) $\mathrm{A}_{2}{ }_{2}$ mode to no longer be suppressed in the resonant Raman spectrum of thinner $\varepsilon$ - 
InSe, as they are in the bulk configuration. A comparison of measured Raman modes, DFTpredicted Raman and IR active modes of few-layered InSe are summarized in Table 1.

Table 1. Summary of Raman spectra of few-layered InSe

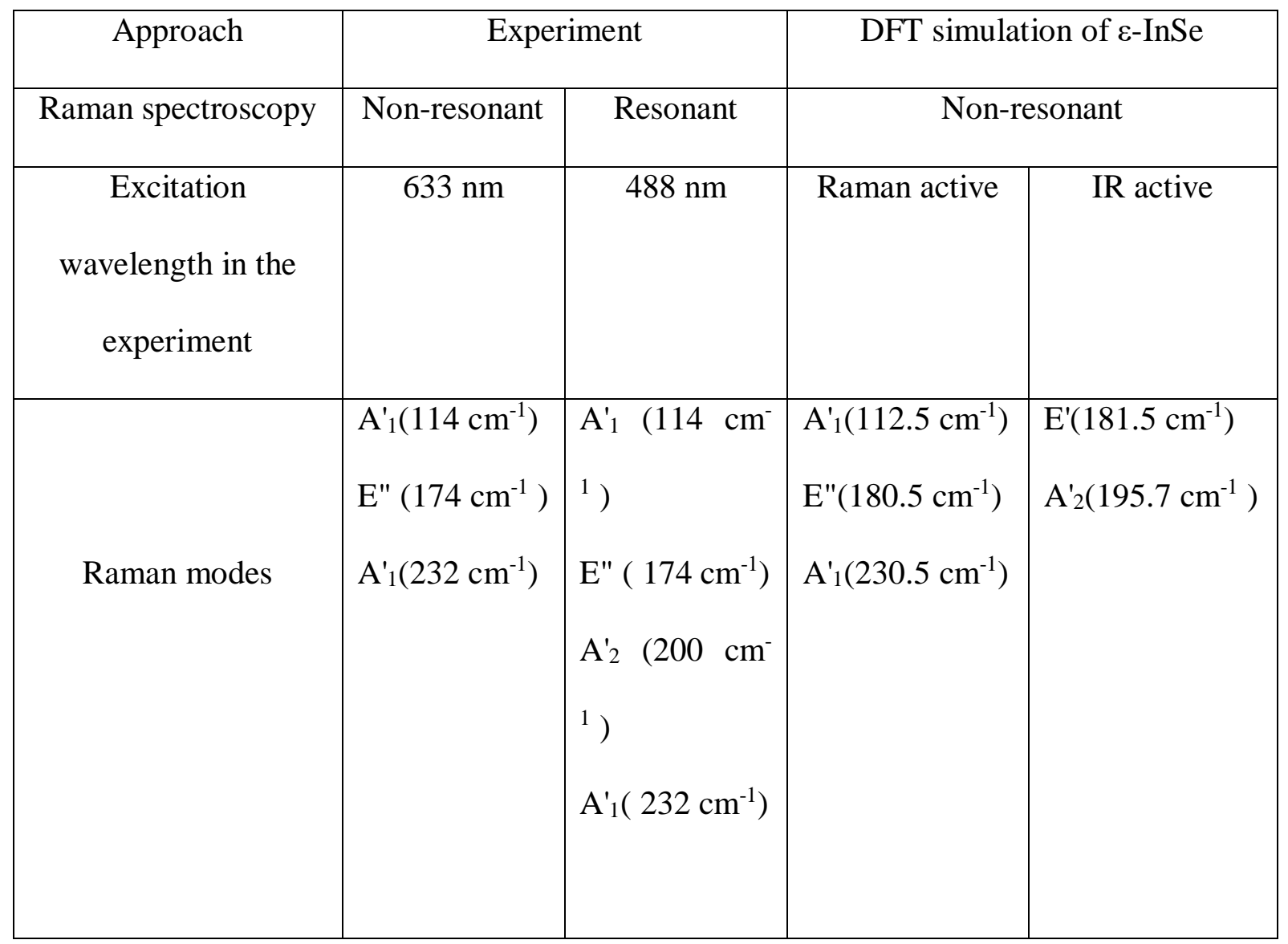

\section{Part 2: Dependence of optoelectronic properties on thickness in multilayer InSe}

In InSe, the absorption coefficient near the fundamental band gap is smaller than that of $\mathrm{E}^{\prime}$ transition. ${ }^{19}$ It is important to look into the thickness dependence of the InSe electronic band structure, particularly for the E' transition, which is the energy difference between the $\mathrm{P}_{\mathrm{x}}, \mathrm{P}_{\mathrm{y}}$-like orbitals to the lowest conduction band,$^{38}$ as shown in Fig. 3(a). To this end, we perform photoluminescence spectroscopy on InSe flakes from 70 to 5 layers with the $488 \mathrm{~nm}$ laser which 
was previously used for resonant Raman characterization. Figure 3(b) shows that as the thickness of InSe flakes is decreased from 70 layers to 8 layers, the peak wavelength of the E' transitioninduced PL is decreased from $510 \mathrm{~nm}$ to $498 \mathrm{~nm}$. This blue shift of the PL peak wavelength indicates that the E' transition energy increases with decreasing flake thickness. However, we are not able to observe the photoluminescence signal for flake thickness below eight layers, either due to the oxidization of InSe at ambient or due to the ash caused by the laser intensity.

We resort to DFT simulations for a rigorous theoretical estimation of the band structure of $\varepsilon$-InSe and its dependence on thickness. For accurate band gap calculations of semiconductors, we perform the density functional linear combination of atomic orbitals (LCAO) using VNL$\mathrm{ATK}^{31}$ with GGA-DFT $+1 / 2^{32}$ method. The spin-orbit coupling is included in our DFT calculations. Details of the simulation parameters are summarized in the computational section. The layered InSe is cleaved from the bulk structure along [001] direction. The DFT-predicted band structure of InSe shows a strong dependence on the number of InSe layers. As we zoom in around the second highest valence bands at the $\Gamma$ point of the Brillouin zone, as shown in Fig. 3(c) with one layered InSe, the spin-orbit coupling has partly lifted the degeneracy of those bands by splitting them. In the absence of spin-orbit coupling, the $\mathrm{p}_{\mathrm{x}, \mathrm{y}}$ states are doubly degenerate at the $\Gamma$-point. The DFT calculation shows that the band gap of InSe is increased from 1.07 to $1.90 \mathrm{eV}$ with a decreasing number of layers, as shown in Fig. 3(d). The DFT-predicted electronic band structure shows that threshold energy of the $\mathrm{E}^{\prime}$ transition is $2.21 \mathrm{eV}$, close to the experimentally observed energy of $2.43 \mathrm{eV}$. As the thickness of the InSe from 20L to monolayer, the DFT predicted E' energy transition is increased from $2.21 \mathrm{eV}$ to $2.65 \mathrm{eV}$, as summarized in Fig. 3(e). 
(a)

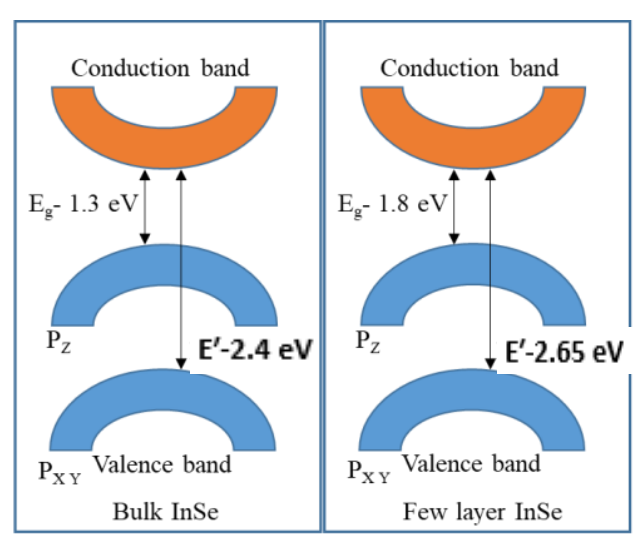

(c)

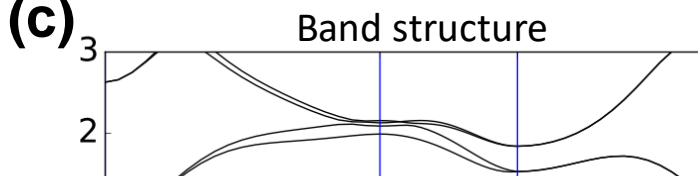

(d)
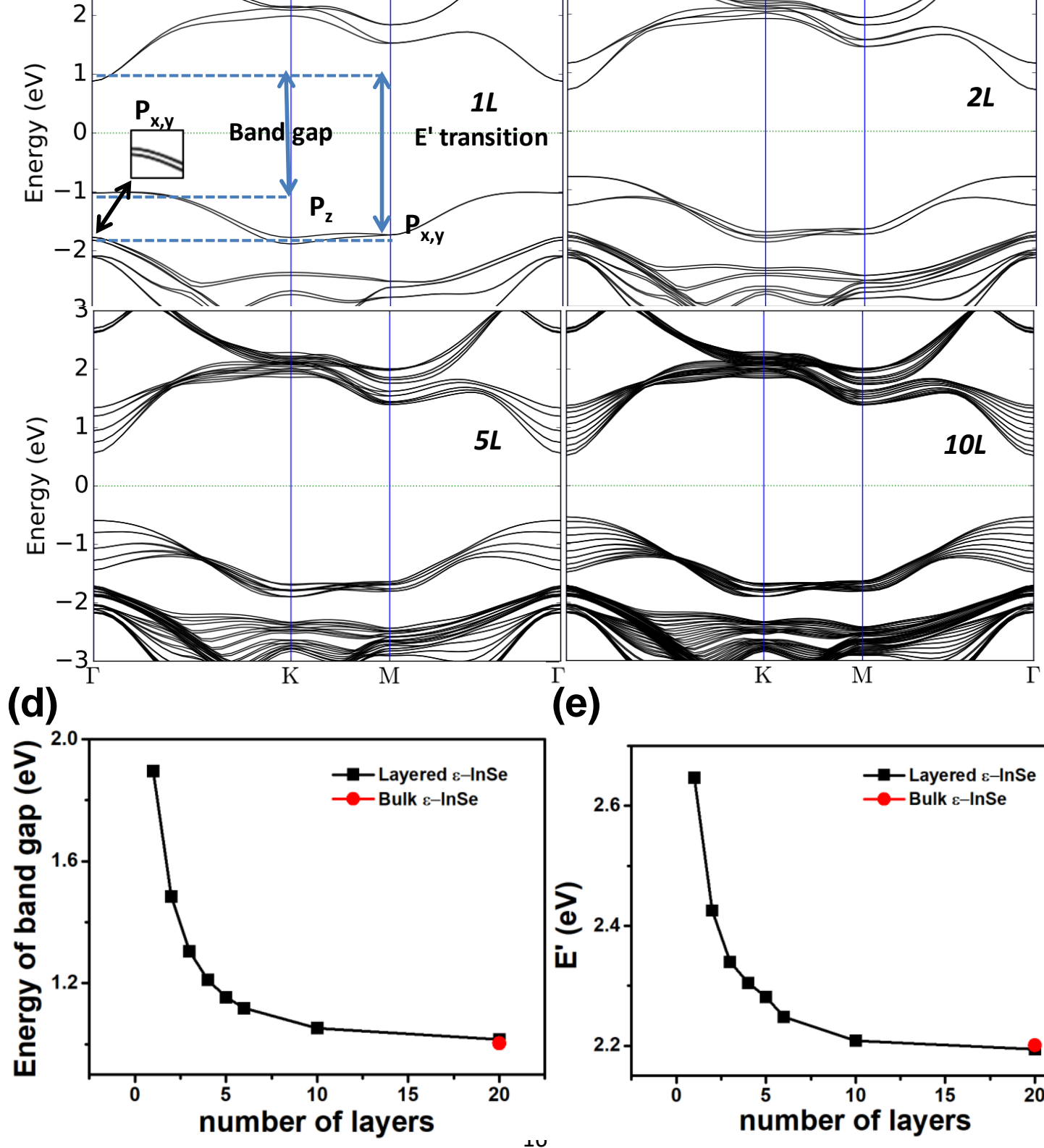

(b)

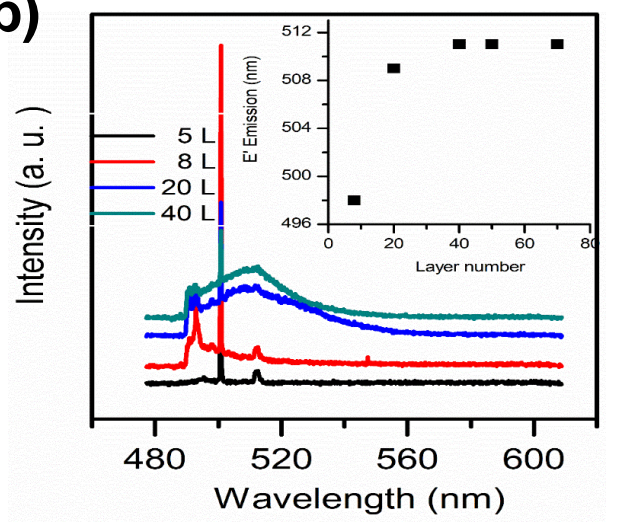

Band structure (e)

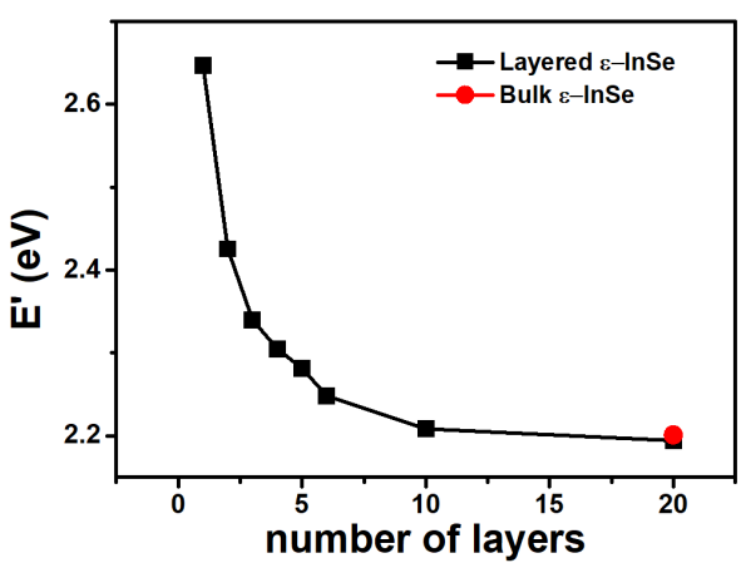


Figure 3. (a) Schematic of electronic band structure of InSe from bulk to few layers. (b) Thickness-dependent PL spectra of InSe Raman spectra and the peak wavelengths are plotted as a function of layer number in the inset. (c) DFT-predicted electronic band structures of $\varepsilon$-InSe from 20 layers to monolayer. (d-e) DFT-predicted energy band gap and E' energy transition as a function of the layer number of $\varepsilon$-InSe.

\section{Part 3: lattice dynamics and photoluminescence of InSe/graphene heterostructure}

To avoid the degradation of InSe, we transfer CVD-grown graphene onto the freshly exfoliated InSe flakes on $\mathrm{SiO}_{2} / \mathrm{Si}$ wafer using a wet-transfer process. The details of the CVD graphene growth and wet transfer process are included in the experimental details in Supplementary Section. After transfer, the InSe flakes covered with graphene show no degradation in ambient environment, in contrast to the area of uncovered InSe. The 8L-InSe and graphene-coated 8L-InSe are characterized using the resonant Raman and PL spectroscopy measurements. During the measurements, a much lower intensity of $488 \mathrm{~nm}$ laser is applied for the uncovered InSe since it is easily be damaged. Significantly, the InSe flakes with the thickness below 8 layers were easily ashed under laser illumination, as shown in the inset of Fig. 4(a). For 8L-InSe covered with graphene, the laser is applied with higher intensity, which causes stronger PL emission in both Raman and PL spectroscopy, as shown in Fig. 4(a-b). The resonant Raman spectroscopy in Fig. 4(a) shows that the $\mathrm{A}_{1}^{\prime}\left(200 \mathrm{~cm}^{-1}\right)$ Raman mode is greatly enhanced by the graphene coating, an illustration of the so-called graphene-enhanced Raman scattering effect. ${ }^{39}$ In addition, the $\mathrm{E}^{\prime}$ transition emission of graphene-coated 8L-InSe can be clearly observed in the photoluminescence spectrum as shown in Fig. 4(b).

We characterized the thickness dependence of graphene coatings on $90 \mathrm{~nm}$-thick InSe flake using resonant Raman spectra (488 nm laser). The measured resonant Raman spectra of graphene coated InSe do not show a significant difference for monolayer, bilayer, and multilayer graphene coatings, as shown in Fig. 4(c). This is opposition to the results of graphene-coated 8L- 
InSe shown in Fig. 4(a) possibly due to the weak absorption of $90 \mathrm{~nm}$ InSe. As it comes to PL spectroscopy of graphene-coated InSe, the $\mathrm{E}^{\prime}$ transition induced PL show strong and stable emission under the monolayer and bilayer graphene coatings. However, the multilayered graphene quenches the PL intensity up to $30 \%$ due to high absorptance of the coating, as shown in Fig. 4(d).

(a)

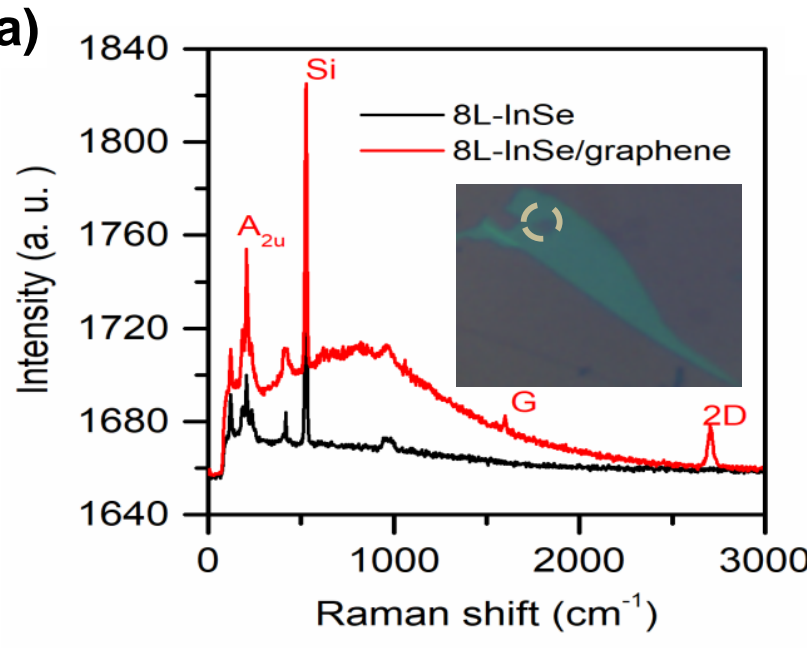

(b)

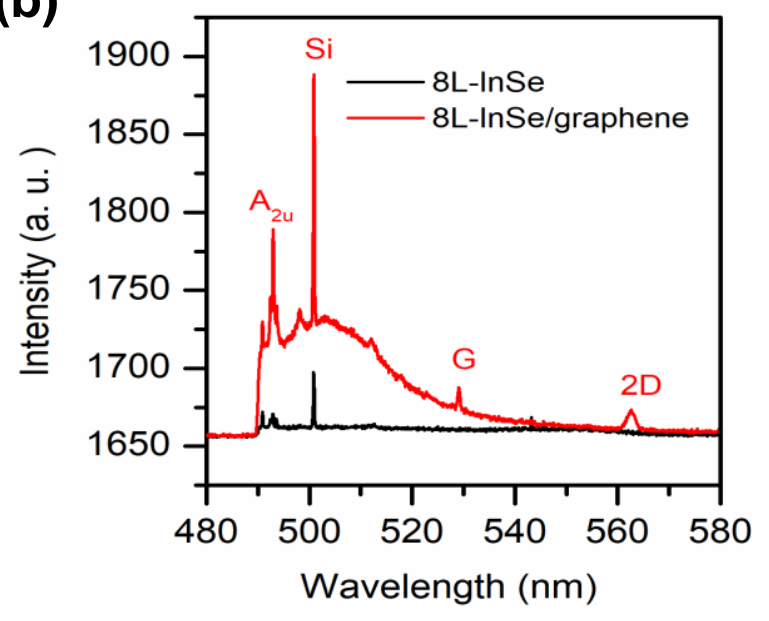

(c)

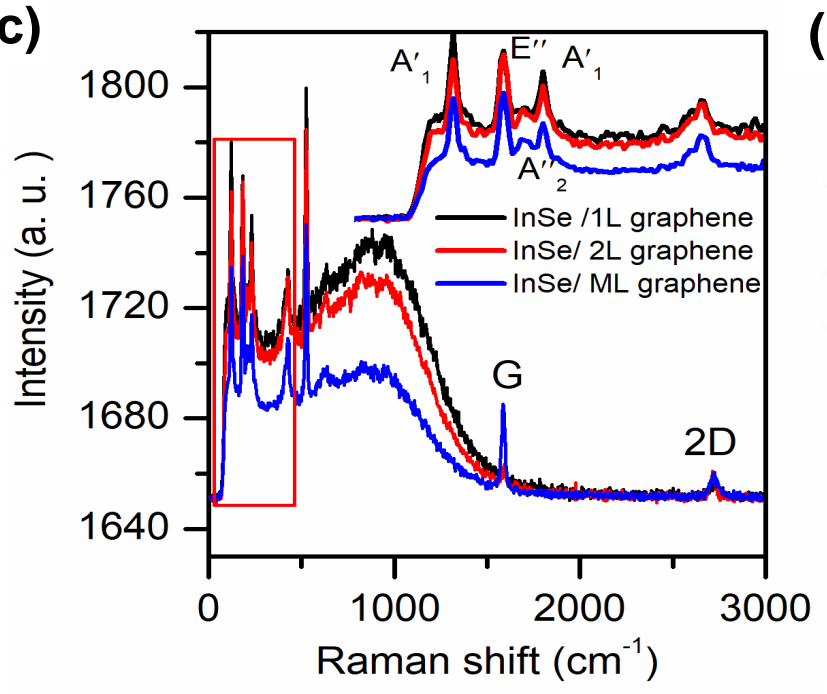

(d)

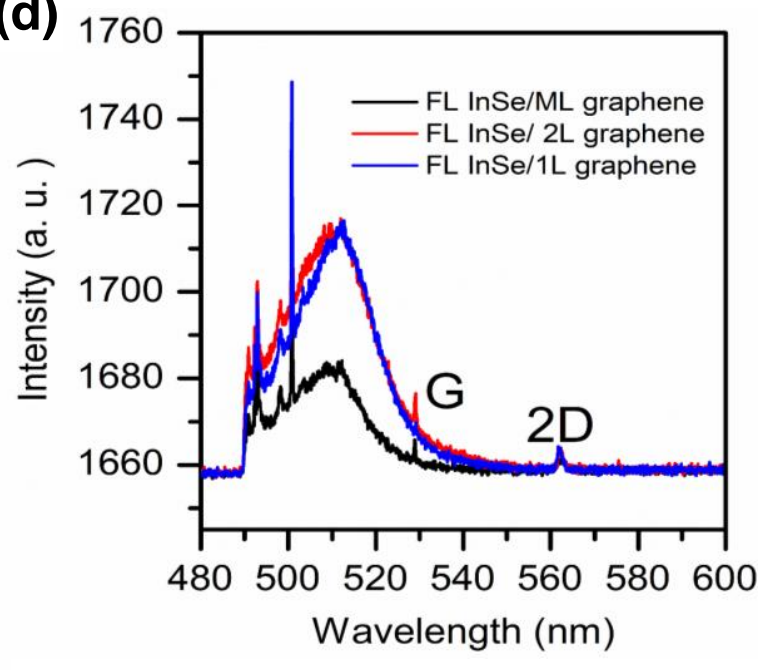

Figure 4. (a-b) Resonant Raman and PL spectra of 8L-InSe with and without graphene coating onto it for $488 \mathrm{~nm}$ laser excitation. An area of uncovered InSe was ashed by the laser shown in the inset. (c) Raman spectra of $90 \mathrm{~nm}$-thick InSe coated with monolayer, bilayer, and multilayer 
graphene coatings. (d) PL spectra of few-layered InSe coated with monolayer, bilayer, and multilayer graphene coatings.

As the graphene is transferred onto the InSe to stabilize it, the graphene/InSe also acts as a heterostructure. Similar, to Van der Wall heterostructures between the graphene and TMDC materials $^{40}$, InSe/graphene heterostructure also exhibited optically induced charge transfer from the InSe to the graphene as shown in Fig. 5(a). In general, the experimental barrier height in graphene-based heterostructures are significantly different than the ones expected by the work function difference of the two materials ${ }^{41}$. This may be due to the unintentional p-doping effect of the CVD graphene film, due to the transfer process, the adsorbed moisture/oxygen and the substrate impurities $^{42}$, which alters the work function of the graphene. In Fig. 5(a), we assume that there is small barrier height between the p-type graphene $(4.8 \mathrm{eV}){ }^{40}$ and $\mathrm{InSe}(4.77 \mathrm{eV})^{43}$. Our DFT simulation results show that as the electron number of the graphene system is larger than that of the neutral system by averagely adding the 0.025 holes into each carbon atom, the graphene G peak will be shifted to a higher wavenumber, which is opposite to that of electrondoped graphene system. As we look into the peak shift in the Raman spectra of graphene coated InSe for all three excitation wavelengths, we find that there is no shift of the graphene $\mathrm{G}$ peak under $633 \mathrm{~nm}$ laser illumination, as shown in Fig. 5(b). As the laser wavelength is decreased to $532 \mathrm{~nm}$ and $488 \mathrm{~nm}$, the graphene $\mathrm{G}$ peak shifts towards higher wavenumber. The shift of the $\mathrm{G}$ peak increases with the laser energy, as shown in Fig. 5(c-d). This indicates that an electron-hole pair is generated by the $E^{\prime}$ transition and the holes are injected into the graphene from the valence bands of the InSe. 
a

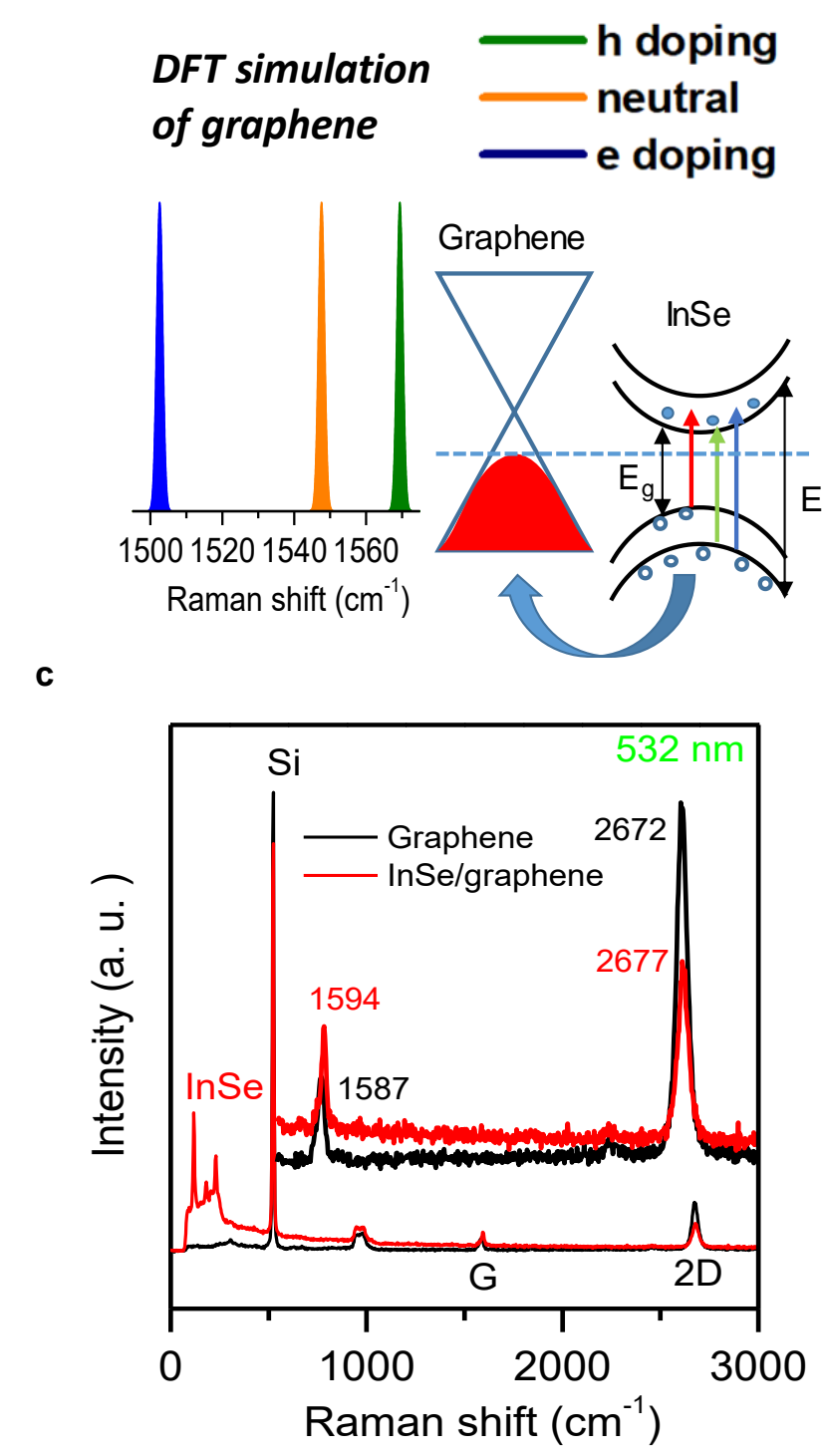

b
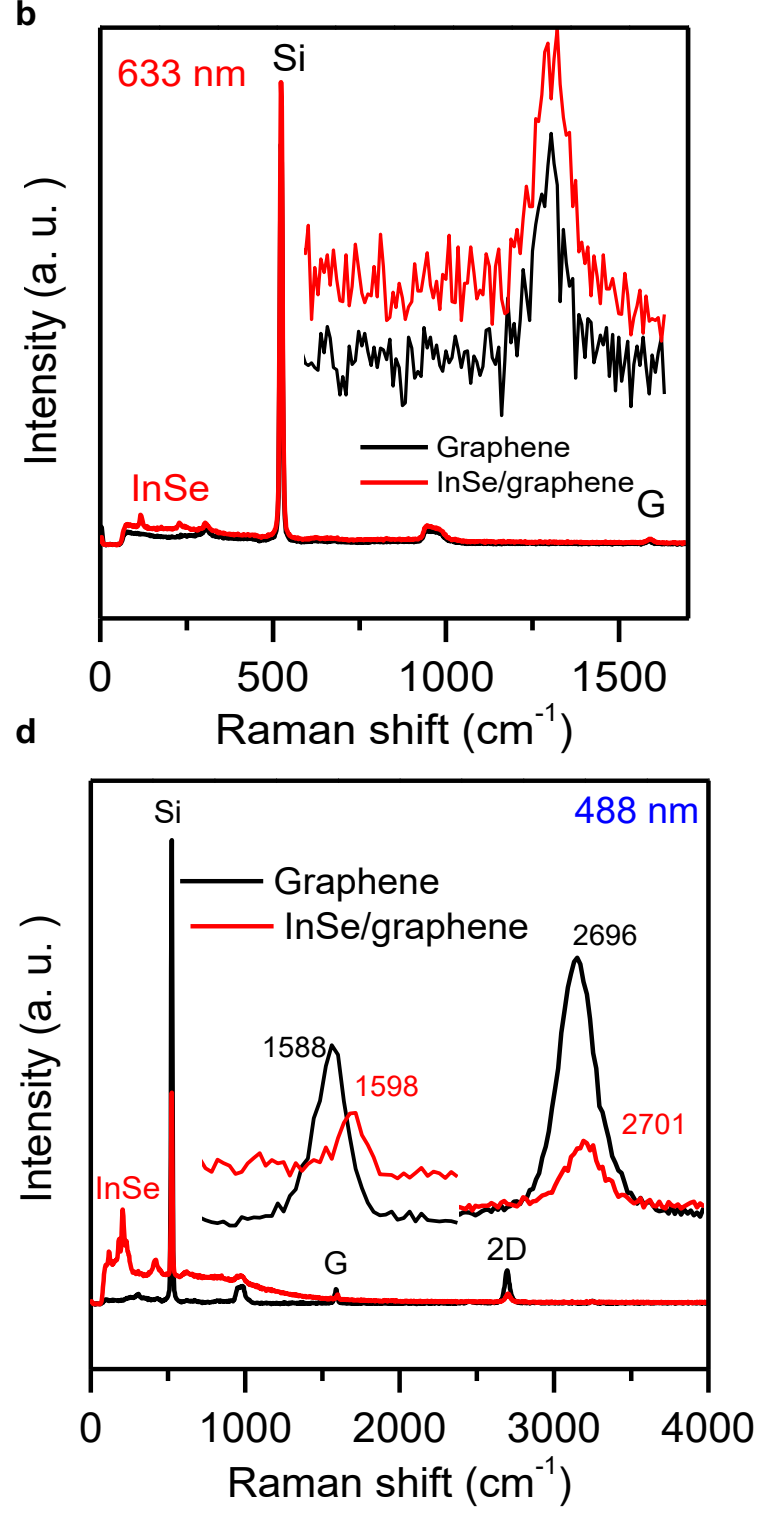

Figure 5. (a) Schematic of charge transfer between graphene and InSe, and the DFT simulation of graphene Raman mode with electron doping, neutral, and hole doping. (b-d) Raman spectra of graphene/InSe heterostructure for three different excitation lasers, including $633 \mathrm{~nm}, 532 \mathrm{~nm}$, and $488 \mathrm{~nm}$, respectively.

\section{Conclusion}

We have performed resonant and non-resonant Raman spectroscopy and density functional theory simulation on hexagonal layered InSe crystals ranging in thickness from bulk to few-layer. We confirmed the crystal structure under the study as $\varepsilon$-phase InSe and demonstrated that the 
measured intensity ratio of Raman peaks in the resonant Raman spectrum provides a way to identify the layer thickness of InSe, which is not revealed in the standard non-resonant Raman spectroscopy. In parallel, the thickness dependent photoluminescence spectra and electronic band structure of InSe are investigated, with emphasis on the $\mathrm{E}^{\prime}$ transition band gap $\sim 2.4 \mathrm{eV}$, whose optical absorption is higher than that of the fundamental band gap. The graphene coated InSe shows improved stability of few-layered InSe and greater stability of E' transition-induced PL under laser illumination. Moreover, electron transfer from the conduction band of InSe to graphene is observed under $488 \mathrm{~nm}$ laser illumination, as indicated by the graphene $\mathrm{G}$ peak shift, which possibly shows a route towards future integrated electronic and optoelectronic devices.

\section{Supporting Information}

Contents of Supporting Information are listed as follows: S. I X-ray diffraction of InSe, S. II DFT predicted frequencies and intensities of phonon modes of $\beta$-InSe, S.III Lattice vibration of phonon modes of $\varepsilon$-InSe, S. IV Electronic band structure of $\beta$-InSe from bulk to monolayer, S. V Raman spectra of few-layered $\varepsilon$-InSe, S.VI AFM topography and height profile of InSe samples, S.VII QuantumEspresso Input files for phonon calculations of bulk $\varepsilon$-InSe.

\section{Data availability}

All of the relevant data is available from the authors. Requests for data and materials should be addressed to Matteo Chiesa.

\section{Acknowledgments}

This work was funded under the Cooperative Agreement between the Khalifa University of Science and Technology, Masdar campus, Abu Dhabi, UAE and the Massachusetts Institute of Technology(MIT), Cambridge, MA, USA, Reference Number FR2017-000001. M.C. 
acknowledges the support of the Arctic Center for Sustainable Energy (ARC), UiT Arctic University of Norway through grant no. 310059.

Competing Interests: The authors declare no competing financial interests.

\section{References:}

(1) Ho, C. H.; Lin, S. L.Optical Properties of the Interband Transitions of Layered Gallium Sulfide. J. Appl. Phys. 2006, 100 (8), 83508.

(2) Yuan, X.; Tang, L.; Liu, S.; Wang, P.; Chen, Z.; Zhang, C.; Liu, Y.; Wang, W.; Zou, Y.; Liu, C.Arrayed van Der Waals Vertical Heterostructures Based on 2D GaSe Grown by Molecular Beam Epitaxy. Nano Lett. 2015, 15 (5), 3571-3577.

(3) Gasanly, N. M.; Aydınlı, A.; Özkan, H.; Kocabaş, C.Temperature Dependence of the First-Order Raman Scattering in GaS Layered Crystals. Solid State Commun. 2000, 116 (3), 147-151.

(4) Tamalampudi, S. R.; Lu, Y.-Y.; Kumar U, R.; Sankar, R.; Liao, C.-D.; Moorthy B, K.; Cheng, C.H.; Chou, F. C.; Chen, Y.-T.High Performance and Bendable Few-Layered InSe Photodetectors with Broad Spectral Response. Nano Lett. 2014, 14 (5), 2800-2806.

(5) Kang, J.; Wells, S. A.; Sangwan, V. K.; Lam, D.; Liu, X.; Luxa, J.; Sofer, Z.; Hersam, M. C.Solution-Based Processing of Optoelectronically Active Indium Selenide. Adv. Mater. 2018, 30 (38), 1802990.

(6) Magorrian, S. J.; Zólyomi, V.; Fal'ko, V. I.Spin-Orbit Coupling, Optical Transitions, and Spin Pumping in Monolayer and Few-Layer InSe. Phys. Rev. B 2017, 96 (19), 195428.

(7) Sucharitakul, S.; Goble, N. J.; Kumar, U. R.; Sankar, R.; Bogorad, Z. A.; Chou, F.-C.; Chen, Y.-T.; Gao, X. P. A.Intrinsic Electron Mobility Exceeding 103 Cm2/(V s) in Multilayer InSe FETs. Nano Lett. 2015, 15 (6), 3815-3819. 
(8) Ho, P.-H.; Chang, Y.-R.; Chu, Y.-C.; Li, M.-K.; Tsai, C.-A.; Wang, W.-H.; Ho, C.-H.; Chen, C.W.; Chiu, P.-W.High-Mobility InSe Transistors: The Role of Surface Oxides. ACS Nano 2017, 11 (7), 7362-7370.

(9) Lee, C.; Yan, H.; Brus, L. E.; Heinz, T. F.; Hone, J.; Ryu, S.Anomalous Lattice Vibrations of Single-and Few-Layer MoS2. ACS Nano 2010, 4 (5), 2695-2700.

(10) Hu, P.; Wen, Z.; Wang, L.; Tan, P.; Xiao, K.Synthesis of Few-Layer GaSe Nanosheets for High Performance Photodetectors. ACS Nano 2012, 6 (7), 5988-5994.

(11) Carlone, C.; Jandl, S.; Shanks, H. R.Optical Phonons and Crystalline Symmetry of InSe. Phys. status solidi 1981, 103 (1), 123-130.

(12) Sánchez-Royo, J. F.; Muñoz-Matutano, G.; Brotons-Gisbert, M.; Martínez-Pastor, J. P.; Segura, A.; Cantarero, A.; Mata, R.; Canet-Ferrer, J.; Tobias, G.; Canadell, E.Electronic Structure, Optical Properties, and Lattice Dynamics in Atomically Thin Indium Selenide Flakes. Nano Res. 2014, 7 (10), 1556-1568.

(13) Politano, A.; Campi, D.; Cattelan, M.; Amara, I.Ben; Jaziri, S.; Mazzotti, A.; Barinov, A.; Gürbulak, B.; Duman, S.; Agnoli, S.Indium Selenide: An Insight into Electronic Band Structure and Surface Excitations. Sci. Rep. 2017, 7 (1), 3445.

(14) Lei, S.; Ge, L.; Najmaei, S.; George, A.; Kappera, R.; Lou, J.; Chhowalla, M.; Yamaguchi, H.; Gupta, G.; Vajtai, R.Evolution of the Electronic Band Structure and Efficient Photo-Detection in Atomic Layers of InSe. ACS Nano 2014, 8 (2), 1263-1272.

(15) Do, D. T.; Mahanti, S. D.; Lai, C. W.Spin Splitting in 2D Monochalcogenide Semiconductors. Sci. Rep. 2015, 5, 17044.

(16) Chevy, A.Improvement of Growth Parameters for Bridgman-Grown InSe Crystals. J. Cryst. Growth 1984, 67 (1), 119-124. 
(17) Efremov, E.V; Ariese, F.; Gooijer, C.Achievements in Resonance Raman Spectroscopy: Review of a Technique with a Distinct Analytical Chemistry Potential. Anal. Chim. Acta 2008, 606 (2), $119-134$.

(18) Favron, A.; Goudreault, F. A.; Gosselin, V.; Groulx, J.; Côté, M.; Leonelli, R.; Germain, J.-F.; Phaneuf-L'Heureux, A.-L.; Francoeur, S.; Martel, R.Second-Order Raman Scattering in Exfoliated Black Phosphorus. Nano Lett. 2018, 18 (2), 1018-1027.

(19) Kuroda, N.; Munakata, I.; Nishina, Y.Exciton Transitions from Spin-Orbit Split off Valence Bands in Layer Compound InSe. Solid State Commun. 1980, 33 (6), 687-691.

(20) Xiao, J.; Zhao, M.; Wang, Y.; Zhang, X.Excitons in Atomically Thin 2D Semiconductors and Their Applications. Nanophotonics 2017, 6 (6), 1309-1328.

(21) Hossain, J.; Julkarnain, M.; Sharif, K. S.; Khan, K. A.Optical Properties of E-Beam Evaporated Indium Selenide (InSe) Thin Films. J. Sci. Res. Reports 2014, 3, 1642-1655.

(22) Shi, L.; Zhou, Q.; Zhao, Y.; Ouyang, Y.; Ling, C.; Li, Q.; Wang, J.Oxidation Mechanism and Protection Strategy of Ultrathin Indium Selenide: Insight from Theory. J. Phys. Chem. Lett. 2017, $8(18), 4368-4373$.

(23) Politano, A.; Chiarello, G.; Samnakay, R.; Liu, G.; Gürbulak, B.; Duman, S.; Balandin, A. A.; Boukhvalov, D. W.The Influence of Chemical Reactivity of Surface Defects on Ambient-Stable InSe-Based Nanodevices. Nanoscale 2016, 8 (16), 8474-8479.

Li, Q.; Zhou, Q.; Shi, L.; Chen, Q.; Wang, J.Recent Advances in Oxidation and Degradation Mechanisms of Ultrathin 2D Materials under Ambient Conditions and Their Passivation Strategies. J. Mater. Chem. A 2019.

Mudd, G. W.; Svatek, S. A.; Hague, L.; Makarovsky, O.; Kudrynskyi, Z. R.; Mellor, C. J.; Beton, P. H.; Eaves, L.; Novoselov, K. S.; Kovalyuk, Z. D.High Broad-band Photoresponsivity of 
Mechanically Formed InSe-Graphene van Der Waals Heterostructures. Adv. Mater. 2015, 27 (25), 3760-3766.

(26) Luo, W.; Cao, Y.; Hu, P.; Cai, K.; Feng, Q.; Yan, F.; Yan, T.; Zhang, X.; Wang, K.Gate Tuning of High-Performance InSe-Based Photodetectors Using Graphene Electrodes. Adv. Opt. Mater. 2015, $3(10), 1418-1423$.

(27)

Chiou, Y.-C.; Olukan, T. A.; Almahri, M. A.; Apostoleris, H.; Chiu, C. H.; Lai, C.-Y.; Lu, J.-Y.; Santos, S.; Almansouri, I.; Chiesa, M.Direct Measurement of the Magnitude of the van Der Waals Interaction of Single and Multilayer Graphene. Langmuir 2018, 34 (41), 12335-12343.

(28) Giannozzi, P.; Andreussi, O.; Brumme, T.; Bunau, O.; Nardelli, M. B.; Calandra, M.; Car, R.; Cavazzoni, C.; Ceresoli, D.; Cococcioni, M.Advanced Capabilities for Materials Modelling with Quantum ESPRESSO. J. Phys. Condens. Matter 2017, 29 (46), 465901.

(29) Perdew, J. P.; Zunger, A.Self-Interaction Correction to Density-Functional Approximations for Many-Electron Systems. Phys. Rev. B 1981, 23 (10), 5048.

(30) Lazzeri, M.; Mauri, F.First-Principles Calculation of Vibrational Raman Spectra in Large Systems: Signature of Small Rings in Crystalline S i O 2. Phys. Rev. Lett. 2003, 90 (3), 36401.

(31) QuantumATK O-2018.06, Synopsys QuantumATK (Https://Www.Synopsys.Com/Silicon/Quantumatk.Html).

(32) Ferreira, L. G.; Marques, M.; Teles, L. K.Slater Half-Occupation Technique Revisited: The LDA1/2 and GGA-1/2 Approaches for Atomic Ionization Energies and Band Gaps in Semiconductors. Aip Adv. 2011, 1 (3), 32119.

(33) Jandl, S.; Carlone, C.Raman Spectrum of Crystalline InSe. Solid State Commun. 1978, 25 (1), 5-8.

(34) Chen, Z.; Biscaras, J.; Shukla, A.A High Performance Graphene/Few-Layer InSe Photo-Detector. Nanoscale 2015, 7 (14), 5981-5986. 
(35) Huang, Y.; Qiao, J.; He, K.; Bliznakov, S.; Sutter, E.; Chen, X.; Luo, D.; Meng, F.; Su, D.; Decker, J.Interaction of Black Phosphorus with Oxygen and Water. Chem. Mater. 2016, 28 (22), 83308339.

(36) Zhao, Q.; Frisenda, R.; Gant, P.; Perez de Lara, D.; Munuera, C.; Garcia-Hernandez, M.; Niu, Y.; Wang, T.; Jie, W.; Castellanos-Gomez, A.Toward Air Stability of Thin GaSe Devices: Avoiding Environmental and Laser-Induced Degradation by Encapsulation. Adv. Funct. Mater. 2018, 28 (47), 1805304.

Trallero-Giner, C.; Cantarero, A.; Cardona, M.One-Phonon Resonant Raman Scattering: Fröhlich Exciton-Phonon Interaction. Phys. Rev. B 1989, 40 (6), 4030.

(38) Camara, M. O. D.; Mauger, A.; Devos, I.Electronic Structure of the Layer Compounds GaSe and InSe in a Tight-Binding Approach. Phys. Rev. B 2002, 65 (12), 125206.

(39) Lai, H.; Xu, F.; Zhang, Y.; Wang, L.Recent Progress on Graphene-Based Substrates for SurfaceEnhanced Raman Scattering Applications. J. Mater. Chem. B 2018, 6 (24), 4008-4028.

(40) Tsuboi, Y.; Wang, F.; Kozawa, D.; Funahashi, K.; Mouri, S.; Miyauchi, Y.; Takenobu, T.; Matsuda, K.Enhanced Photovoltaic Performances of Graphene/Si Solar Cells by Insertion of a MoS 2 Thin Film. Nanoscale 2015, 7 (34), 14476-14482.

(41) Kalita, G.; Dzulsyahmi Shaarin, M.; Paudel, B.; Mahyavanshi, R.; Tanemura, M.Temperature Dependent Diode and Photovoltaic Characteristics of Graphene-GaN Heterojunction. Appl. Phys. Lett. 2017, $111(1), 13504$.

(42) Zhan, D.; Sun, L.; Ni, Z. H.; Liu, L.; Fan, X. F.; Wang, Y.; Yu, T.; Lam, Y. M.; Huang, W.; Shen, Z. X.FeCl3-Based Few-Layer Graphene Intercalation Compounds: Single Linear Dispersion Electronic Band Structure and Strong Charge Transfer Doping. Adv. Funct. Mater. 2010, 20 (20), 3504-3509. 
(43) Sang, D. K.; Wang, H.; Qiu, M.; Cao, R.; Guo, Z.; Zhao, J.; Li, Y.; Xiao, Q.; Fan, D.; Zhang, H.Two Dimensional $\beta$-InSe with Layer-Dependent Properties: Band Alignment, Work Function and Optical Properties. Nanomaterials 2019, 9 (1), 82. 
TOC image:
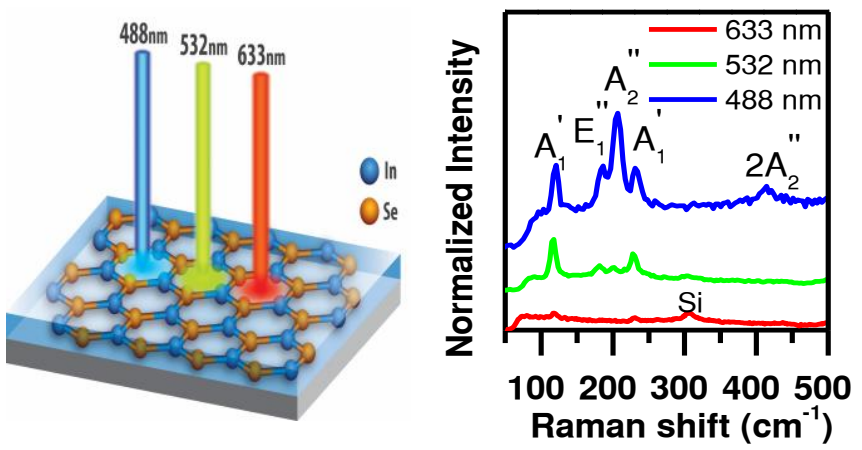